\title{
Understanding the epidemiology of MRSA in Europe: do we need to think outside the box?
}

\author{
M.A. Borg ${ }^{a, *}$, L. Camilleri ${ }^{b}$, B. Waisfisz ${ }^{c}$ \\ a Infection Control Unit, Mater Dei Hospital, Msida, Malta \\ ${ }^{\mathrm{b}}$ Department of Statistics and Operations Research, University of Malta, Msida, Malta \\ 'ITIM Focus, The Hague, The Netherlands
}

\section{A R T I C L E I N F O}

\section{Article history:}

Received 26 January 2012

Accepted 1 May 2012

Available online 12 June 2012

\section{Keywords:}

Culture

Hofstede

Infection

\section{S U M M A R Y}

Background: The epidemiology of meticillin-resistant Staphylococcus aureus (MRSA) infections, using bacteraemia as a marker, shows a striking geographical pattern in Europe. The prevalence of MRSA is low in Northern European countries, increases into central Europe and reaches its highest levels in the Mediterranean region. This has been attributed to varying levels of implementation of infection control and antibiotic stewardship (ICAS) programmes, but reasons for this variation have not been clearly established.

Aim: To investigate the possible impact of national cultural dimensions on the epidemiology of MRSA in Europe.

Methods: Median proportions of MRSA bacteraemia were sourced for countries participating in the EARS-Net surveillance network in 2010, and correlated with the national cultural dimension scores of Hofstede et al.

Findings: Significant associations were identified between MRSA proportions and the cultural constructs of uncertainty avoidance (UAI), masculinity (MAS) and power distance. Multiple regression models found significant associations for UAI, MAS and short-term orientation $\left(R^{2}\right.$ adjusted $\left.=0.475 ; P<0.001\right)$. The model was found to be predictive of MRSA trends identified in several European countries between 2006 and 2010.

Conclusion: Implementation of ICAS programmes often requires behavioural change. Cultural dimensions appear to be key factors affecting perceptions and values among healthcare workers, which in turn are critical for compliance and uptake. Customizing ICAS initiatives to reflect the local cultural background may improve their chances of success.

(C) 2012 The Healthcare Infection Society. Published by Elsevier Ltd. All rights reserved.

\section{Introduction}

Bloodstream infections (BSI) caused by meticillin-resistant Staphylococcus aureus (MRSA) constitute a major challenge to healthcare institutions, particularly in terms of associated mortality. ${ }^{1}$ A significant reduction in MRSA BSI has been shown

\footnotetext{
* Corresponding author. Address: Infection Control Unit, Mater Dei Hospital, Msida, Malta. Tel.: +356 2545 4528; fax: +356 25457474.

E-mail address: michael.a.borg@gov.mt (M.A. Borg).
}

to be achievable through effective infection control and antibiotic stewardship (ICAS) interventions within hospitals. ${ }^{2}$ As a result, mandatory surveillance of MRSA BSI coupled with reduction targets has been introduced in some countries. ${ }^{3}$ Improvement in MRSA BSI incidence is used to monitor the effectiveness of ICAS programmes. ${ }^{4}$ National MRSA proportions have been shown to correlate well with incidence, and have been used to calculate overall MRSA incidence at country level. ${ }^{5}$ For more than a decade, comparable surveillance of MRSA proportions in blood cultures has been ongoing in the 
majority of European countries through the European Antimicrobial Resistance Surveillance System (EARSS). The results from the EARSS network, now EARS-Net, show a consistent geographical pattern. The prevalence of MRSA is low (at times practically absent) in almost all Northern European countries, increases into central Europe and reaches its highest levels in the Mediterranean region. ${ }^{6}$

Similar geographical variation exists in ambulatory care antibiotic consumption within the same region. ${ }^{7}$ This has been ascribed to be linked to national cultural differences. ${ }^{8,9}$ Hofstede's model of cultural dimensions is one of the most accepted approaches for analysis of cultural differences between countries, with more than 800 citations in peerreviewed journals. It defines culture as the collective programming of the mind that distinguishes the members of one group or category of people from another. Hofstede formulated a model which proposes that national cultures vary along consistent, fundamental dimensions which can be grouped and scored as specific constructs: power distance (PDI), individualism (IDV), masculinity (MAS) and uncertainty avoidance (UAI). Later, in collaboration with other colleagues, he identified two more dimensions following the analysis of diverse databanks: long-term vs short-term orientation (LTO) and indulgence vs restraint (IVR) (Box 1). ${ }^{10}$

\section{Methods}

The MRSA proportions reported in the 2010 annual epidemiological report of EARS-Net were used in this study. ${ }^{11}$ EARSNet calculated the proportion for each hospital by using the number of $S$. aureus blood culture isolates that showed oxacillin resistance as the numerator, and the total number of S. aureus bacteraemias as the denominator, both on a first per patient basis. The median national proportion was established for each participating country. Hofstede scores for PDI, IDV, MAS, UAI, LTO and IVR were identified for the countries in the EARS-Net surveillance network. These are freely available for research purposes from Hofstede's website and publications. ${ }^{12}$ EARS-Net countries for which Hofstede scores were not available were excluded from further analysis. Pearson correlation was performed between the median hospital MRSA proportion for each country and the corresponding national Hofstede scores. Multiple regression models were built through the forward technique using each country's median MRSA proportion as the dependant variable. Adequacy of the models was confirmed through analysis of variance, Durbin-Watson test for autocorrelation and colinearity diagnostics. Analyses were performed using Statistical Package for the Social Sciences Version 20 (SPSS Inc., Chicago, IL, USA) assuming a 0.05 level of significance.

EARS-Net countries with a minimum of 10 participating hospitals that reported MRSA median proportions of $15 \%$ or more during 2010 were identified. MRSA proportions reported by every hospital in each of these countries were also retrieved for the years 2006-2009, again from the respective EARS-Net annual reports. Generalized linear regression models were used to identify significant changes in the MRSA proportions over the five-year period for each country. Where the distribution of proportions reported by hospitals in a country was symmetrical, a normal distribution was assumed; where the distribution of proportions was right skewed, a Gamma distribution was assumed.

\section{Results}

MRSA proportions for 2010 were available for 26 European countries participating in EARS-Net. Median hospital proportions varied from $0 \%$ in Denmark, Estonia, the Netherlands, Norway and Sweden to $55.6 \%$ in Malta. Hofstede scores were available for all EARS-Net countries except Cyprus and Iceland; these two countries were therefore excluded from further analysis (Table I). On univariate analysis, MRSA proportions were found to be significantly associated with UAI, MAS and PDI (Table II). On multiple regression analysis, MRSA proportions were found to be significantly associated with UAI, MAS and LTO. The model explained $47.5 \%$ of the variation in national MRSA proportions within Europe $\left(R^{2}\right.$ adjusted $=0.475$, $P<0.001$ ) (Table II).

In 2010, 11 countries reported MRSA proportions of $15 \%$ or more from more than 10 hospitals (Figure 1). Following regression analysis, most of these countries did not show any significant trends between 2006 and 2010. However, significant year-on-year reductions in MRSA were recorded in France [beta coefficient $(B)=-0.0107, P=0.001]$, Ireland $(B=-0.0358$, $P<0.001)$ and the UK $(B=-0.0238, P<0.001)$.

\section{Discussion}

A number of studies have attempted to elucidate reasons for the variation in MRSA rates in Europe. Hansen et al. concluded that countries with decreasing MRSA proportions showed stricter ICAS implementation within hospitals. ${ }^{13}$ The ARPAC

\section{Box 1}

Hofstede definitions for the six cultural dimensions (adapted from http://www.geerthofstede.nl).

Power distance (PDI) relates to the extent to which the less powerful members of organizations and institutions accept and expect that power is distributed unequally. It suggests that a society's level of inequality is endorsed by the followers as much as by the leaders.

Uncertainty avoidance (UAI) indicates the extent to which a society tolerates uncertainty and ambiguity, and shows how comfortable its members feel in unstructured situations which are novel, unknown, surprising or different from usual.

Individualism (IND) is the degree to which individuals are integrated into tight groups (collectivist) or loose groups (individualist). Masculinity (MAS) refers to the distribution of roles between the genders. In masculine cultures, ego needs, assertiveness and success are emphasized. In feminine cultures, caring for the weak and quality of life are more important.

Long-term orientation (LTO) reflects long-term pragmatic attitudes vs short-term normative attitudes. Cultures scoring high on this dimension show emphasis on future rewards, in particular saving, persistence and adapting to changing circumstances.

Indulgence (IVR) reflects societies that allow people to enjoy life and have fun vs societies where restraint is emphasized. 
Table I

Median meticillin-resistant Staphylococcus aureus (MRSA) proportions from hospitals in 26 European countries as reported by the EARS-Net network for 2010 together with the Hofstede dimensional scores for the respective countries

\begin{tabular}{|c|c|c|c|c|c|c|c|c|}
\hline \multirow{3}{*}{$\begin{array}{l}\text { Country } \\
\text { Austria }\end{array}$} & \multicolumn{2}{|c|}{ EARS-Net results } & \multicolumn{6}{|c|}{ Hofstede country scores } \\
\hline & \multirow{2}{*}{$\begin{array}{c}\text { Hospitals } \\
107\end{array}$} & \multirow{2}{*}{$\begin{array}{c}\begin{array}{c}\text { Median } \\
\text { MRSA (\%) }\end{array} \\
3.6\end{array}$} & \multicolumn{3}{|c|}{ PDI IDV MAS } & UAI & \multicolumn{2}{|c|}{ LTO IVR } \\
\hline & & & 11 & 55 & 79 & 70 & 60 & 63 \\
\hline Belgium & 36 & 19.8 & 65 & 75 & 54 & 94 & 82 & 57 \\
\hline Bulgaria & 18 & 17.8 & 70 & 30 & 40 & 85 & 69 & 16 \\
\hline Czech Republic & 66 & 13.3 & 57 & 58 & 57 & 74 & 70 & 29 \\
\hline Denmark & 51 & 0 & 18 & 74 & 16 & 23 & 35 & 70 \\
\hline Estonia & 19 & 0 & 40 & 60 & 30 & 60 & 82 & 16 \\
\hline Finland & & & 33 & 63 & 26 & 59 & 38 & 57 \\
\hline France & 57 & 21.9 & 68 & 71 & 43 & 86 & 63 & 48 \\
\hline Gern & 128 & 19.1 & 35 & 67 & 66 & 65 & 83 & 40 \\
\hline Greece & 45 & 39.8 & 60 & 35 & 57 & 112 & 45 & 50 \\
\hline Hungary & 44 & 25.3 & 46 & 80 & 88 & 82 & 58 & 31 \\
\hline Irelan & 50 & 23.8 & 28 & 70 & 68 & 35 & 24 & 65 \\
\hline Italy & 44 & 36.8 & 50 & 76 & 70 & 75 & 61 & 30 \\
\hline & & & 44 & 70 & 9 & 63 & 69 & 13 \\
\hline Lith & 16 & 5.3 & 42 & 60 & 19 & 65 & 82 & 16 \\
\hline Luxembou & 6 & 15 & 40 & 60 & 50 & 70 & 64 & 56 \\
\hline Malta & 3 & 55.6 & 56 & 59 & 47 & 96 & 47 & 66 \\
\hline Nether & 56 & 0 & 38 & 80 & 14 & 53 & 67 & 68 \\
\hline Nor & 39 & 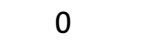 & 31 & 69 & 8 & 50 & 35 & 55 \\
\hline Poland & 49 & 11.1 & 68 & 60 & 64 & 93 & 38 & 29 \\
\hline Portugal & 23 & 49.1 & 63 & 27 & 31 & 104 & 28 & 33 \\
\hline Romania & 6 & 37.1 & 90 & 30 & 42 & 90 & 52 & 20 \\
\hline Slovenia & 15 & 10 & 71 & 27 & 19 & 88 & 49 & 48 \\
\hline Spain & 41 & 24.2 & 57 & 51 & 42 & 86 & 48 & 44 \\
\hline Sweden & 59 & 0 & 31 & 71 & 5 & 29 & 53 & 78 \\
\hline UK & 93 & 27.3 & 35 & 89 & 66 & 35 & 51 & 69 \\
\hline
\end{tabular}

PDI, power distance; UAI, uncertainty avoidance; IND, individualism; MAS, masculinity; LTO, long-term vs short-term orientation; IVR, indulgence vs restraint.

study identified correlations between MRSA prevalence and antibiotic consumption, use of alcohol hand rub and compliance with isolation policies. ${ }^{14}$ Nevertheless, these studies did not explain why these ICAS measures have not been implemented equally effectively across all European countries. This is especially relevant considering that the European Union has made a concerted effort to reduce healthcare-associated infections (HAl) caused by resistant organisms such as MRSA over the past decade. ${ }^{15}$ During this time, numerous change tools have become available for achieving better hand hygiene compliance, ${ }^{16}$ antibiotic stewardship, ${ }^{17}$ and prevention of device-associated bacteraemia. ${ }^{18}$ Moreover, there is clear evidence of the cost-effectiveness of ICAS, both in financial terms and in terms of patient safety. ${ }^{19}$ However, despite the potential benefits to be gained from HAl reduction, few European countries have achieved significant improvement in MRSA BSI.

Nicol et al. proposed a model for healthcare worker (HCW) compliance with ICAS practices such as hand hygiene, based on the theory of planned behaviour. ${ }^{20}$ They suggested that satisfactory behaviour must be preceded by predisposition. The key to success is risk appraisal by HCWs, who need to genuinely
Table II

Individual correlation and multiple regression analysis between national Hofstede cultural dimension scores and 2010 meticillinresistant Staphylococcus aureus (MRSA) proportions reported by countries participating in EARS-Net

\begin{tabular}{lcc}
\hline Cultural dimension & Correlation coefficient & $P$ \\
\hline Univariate analysis & & \\
PDI & 0.515 & 0.007 \\
UAI & 0.603 & 0.001 \\
MAS & 0.461 & 0.018 \\
IND & -0.314 & 0.119 \\
LTO & -0.237 & 0.243 \\
IVR & -0.078 & 0.703 \\
Multiple regression & & \\
Constant & -1.551 & \\
UAI & 0.365 & 0.002 \\
MAS & 0.214 & 0.05 \\
LTO & -0.281 & 0.05 \\
Adjusted $R^{2}$ & 0.475 & \\
$F$ ratio & 8.53 & \\
$P$ & 0.001 & \\
\hline
\end{tabular}

PDI, power distance; UAI, uncertainty avoidance; IND, individualism; MAS, masculinity; LTO, long-term vs short-term orientation; IVR, indulgence vs restraint.

accept and comprehend the risks and impact of HAls before performing the necessary ICAS interventions to mitigate them. It follows that one of the most important elements that drives behaviour in humans - values and perceptions - could have a major impact on ICAS-related practice and outcomes. The predictive model identified UAI as the strongest cultural dimension related to the prevalence of MRSA. Hofstede emphasized that uncertainty avoidance is not the same as risk avoidance. Indeed, people in high UAI countries are reported to be very tolerant of familiar risk and prone to management by crisis. HCWs in these countries are therefore more likely to underestimate HAls which they encounter on a regular daily basis, and tolerate risk activities that predispose to them. It is more challenging to instill ownership of HAl prevention where the problem is regarded as a hospital-wide issue, rather than one in which the individual HCW can make a direct impact. There is a greater likelihood of non-compliance of key ICAS interventions, such as hand hygiene, which require extra effort or time, and may be perceived as increasing work-related pressures. Indeed, the avoidance of stress is a major element of UAl cultures. The authors believe that it is not coincidental that the five European countries with the highest MRSA proportions all possess very high UAl scores. However, UAI was not the only component of the predictive model, which also incorporated MAS and LTO. MAS cultures are highly assertive, decisive and driven by precise targets. As a result, they tend to be less effective than more feminine cultures in activities that require a caring attitude. Furthermore, it is logical to extrapolate that administrators in cultures that score low in LTO would also be less amenable to support and fund ICAS programmes which may need considerable investment over several years before showing reductions in infection rates.

The regression analysis showed that the greatest reduction in MRSA proportions in Europe over the past five years was achieved in the UK and Ireland. This confirms previous 


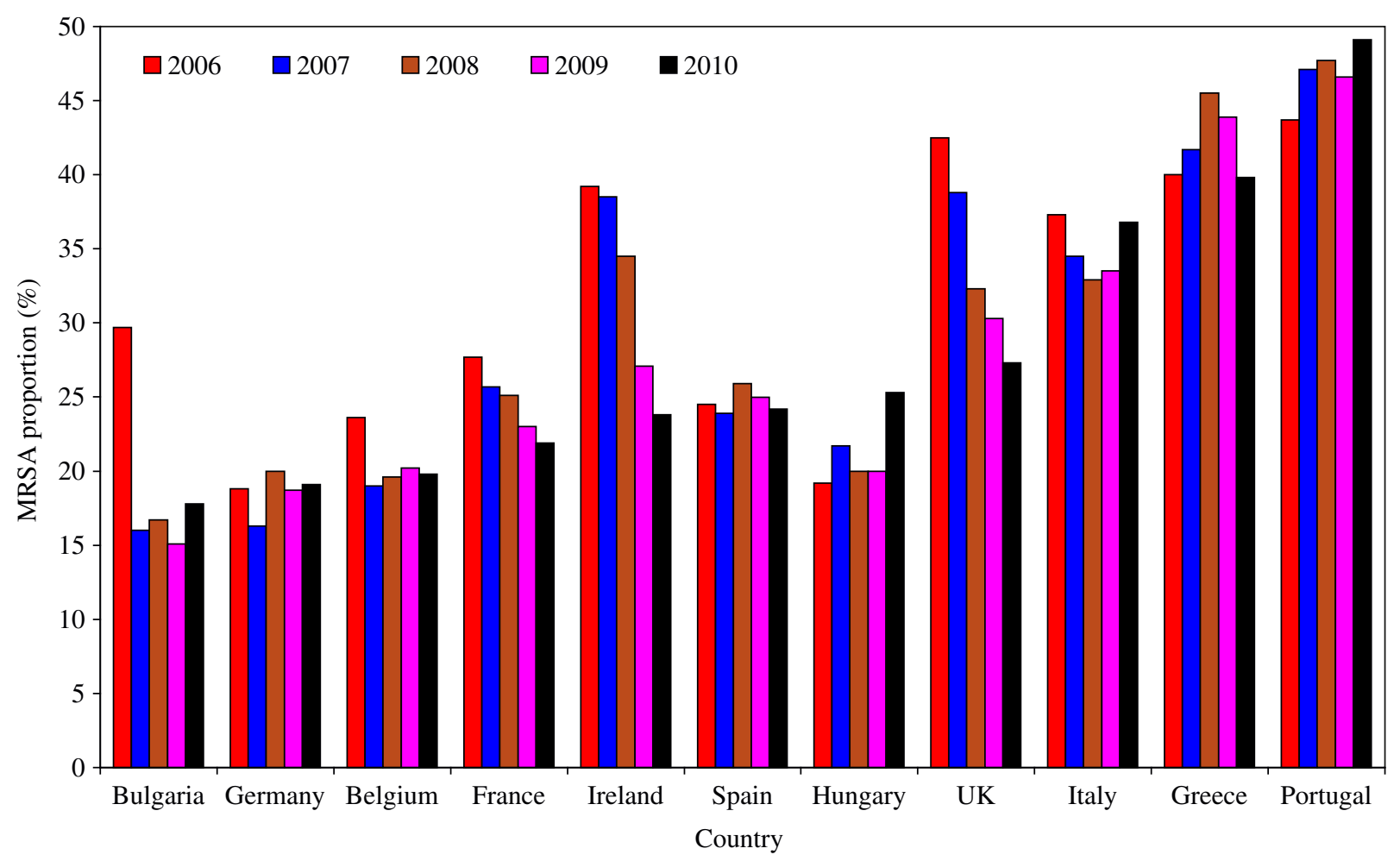

Figure 1. Five-year trends for EARS-Net countries reporting median meticillin-resistant Staphylococcus aureus (MRSA) proportions of 15\% or more in 2010 from at least 10 hospitals.

publications from these countries, highlighting the rapidity and extent by which MRSA levels have decreased. ${ }^{21}$ Improvement was achieved in both intensive care settings and general wards. Both countries scored low for UAI but high for MAS. Targets are extremely effective in such a cultural mindset, which explains the rapid change achieved when MRSA and Clostridium difficile reduction became genuinely important national and institutional goals. ${ }^{3}$ However, a significant reduction in MRSA has also been evident in France. This might appear incongruent as France shows substantially higher UAl scores than the UK and Ireland, as well as lower MAS scores, especially in professional socio-economic classes. However, the improvement trends in this country show somewhat different characteristics to those in the British Isles, not least in the extent and speed of reduction achieved. Jarlier et al. reported a concerted campaign in France, starting in 1993, based primarily on guidelines, barrier precautions and isolation. ${ }^{22}$ Improvement over subsequent years was modest, but increased following a central ministerial initiative in 2001 that implemented five reportable national quality indicators to benchmark hospital performance. ${ }^{23}$ Improvement was sharpest in intensive care units and less rapid in general wards, especially in medical departments where the reduction before 2001 was negligible. ${ }^{22}$ Malta and Italy have also reported significant improvement in MRSA incidence in intensive care that was not evident in general wards. In high UAI backgrounds, change may be more difficult to implement in the heterogeneous environments found in general wards as opposed to intensive care environments, where nurses are normally assigned to individual patients and may feel a greater personal ownership for the outcome.

The data used for this study were derived from the extensive EARS-Net network and its standardized surveillance protocol covering more than 1000 European hospitals in 26 countries. Calculating median MRSA proportions based on individual participating hospitals, rather than the overall country mean, reduces the risk of possible bias from larger hospitals reporting inordinate numbers of isolates, especially in smaller countries. However, this study has a number of limitations. First, the extent of national coverage by EARS-Net laboratories is far from uniform across Europe, being quite low in some Eastern European countries. The EARS-Net protocol does not differentiate between healthcare or community acquisition. Community-acquired MRSA is becoming increasingly important in the European landscape. ${ }^{24}$ Second, some publications have attributed reduction within individual hospitals to MRSA strain variation over time. ${ }^{25}$ Nevertheless, the UK and French experiences clearly demonstrate the utility of MRSA bacteraemia as a marker for the effectiveness of hospital ICAS implementation. Third, Hofstede scores originated from studies in the general population, rather than specifically among healthcare professionals and administrators.

The model used in this study was able to account for almost half of the variance in European MRSA proportions solely on the basis of national cultural dimensions. Provided that the identified association is causal in nature, it introduces a novel parameter in the aetiology of HAl. The regression model shows concomitant variation at a remarkable level of statistical significance. Temporal priority is also present, with national cultural dimensions predating modern medicine by centuries. However, causality also requires elimination of plausible alternatives and confounders. Cultural dimensions have been shown to be associated with inappropriate antibiotic use in European ambulatory care. ${ }^{8}$ Antibiotic misuse in such settings can predispose to community acquisition of MRSA and its 
introduction into hospital on admission of the patient. Nevertheless, the UK experience, where the decline in MRSA started well before admission screening was introduced, suggests that this is unlikely to be a major factor. ${ }^{21}$ Moreover, even if confounding were to be a factor, the present findings would still indicate that some determinant of societal characteristics also contributes to the observed variation in European MRSA rates.

Whilst potentially controversial, a causal association between cultural characteristics and MRSA rates should not be totally surprising since ICAS implementation is ultimately behavioural science: the need for culture change to obtain lasting reduction in $\mathrm{HAl}$ has been emphasized repeatedly. ${ }^{26}$ Culture is often regarded as a nebulous concept, but the present study suggests that clearly defined and measurable national traits impact heavily on patient safety and quality of care initiatives. For ICAS programmes to be effective over the whole range of hospitals in a country, rather than just in individual institutions, they may need to overcome core values that have developed over centuries (Hofstede defines them as mental software). These differ significantly between countries. The bulk of ICAS implementation literature originates from and has been validated in Anglo-Saxon countries, all of which are characterized by low UAI and high MAS. It is not surprising that they focus heavily on surveillance and audit together with collaborative teamwork as components of a plan-do-study-act cycle. ${ }^{27}$ However, audits are specifically meant to identify suboptimal practice and are, by their very nature, major generators of uncertainty. In strong UAI cultures, they can elicit severe discomfort amongst HCWs, further increasing the daily individual stress experienced by people in these countries. This may increase the tendency to ignore them. Worse, they may trigger a counter-productive antagonism, especially where MAS is also high. Indeed, a large cohort of HCWs from eight Mediterranean countries felt that audit and feedback was the least important intervention to improve hand hygiene in their hospitals. ${ }^{28}$ High UAl cultures are also characterized by respect for those with knowledge, reflected in deference for titles and degrees. Furthermore, there is a strong correlation between UAI and PDI among European countries, which was evident in the zero order coefficients of the model used in this study. In such environments, it can be a challenge to convince administrators to adopt a no-blame approach to audit results, achieve equitable collaboration between different healthcare professionals (especially doctors with nurses), or foster a genuine culture of collegial reminders such as in instances of hand hygiene non-compliance. ${ }^{29}$ These have been the cornerstone of many successful infection prevention initiatives in the USA, UK and Northern Europe. ${ }^{30}$ To ensure maximal uptake and impact, it is therefore crucial that ICAS interventions transposed from a different background are adapted to the local culture.

Culture is, of course, not the only factor affecting the epidemiology of HAl. However, the association identified in the present study offers a new insight into our understanding of infection prevention. It would explain the lack of improvement in high MRSA endemic countries with conducive combinations of cultural traits. More research is clearly required into these preliminary findings. Studies that focus on ICAS parameters, rather than surrogate markers, and correlate behaviour and compliance with cultural dimensions of HCW cohorts are needed. Better understanding of cultural influences may help European infection control professionals achieve meaningful behavioural change and reduce $\mathrm{HAl}$ in their own particular settings.

\section{Acknowledgements}

The authors wish to thank Jennie Wilson and Annalisa Pantosti for feedback on MRSA trends in England and Italy, respectively.

\section{Conflict of interest statement}

None declared.

\section{Funding sources}

None.

\section{References}

1. de Kraker ME, Wolkewitz M, Davey PG, et al. Clinical impact of antimicrobial resistance in European hospitals: excess mortality and length of hospital stay related to methicillin-resistant Staphylococcus aureus bloodstream infections. Antimicrob Agents Chemother 2011;55:1598-1605.

2. Thompson DS, Workman R, Strutt M. Decline in the rates of meticillin-resistant Staphylococcus aureus acquisition and bacteraemia in a general intensive care unit between 1996 and 2008. J Hosp Infect 2009;71:314-319.

3. Duerden BI. Controlling healthcare-associated infections in the NHS. Clin Med 2008;8:140-143.

4. Duerden BI. Responsibility for managing healthcare-associated infections: where does the buck stop? J Hosp Infect 2009;73: 414-417.

5. Davey P, Sneddon J, Nathwani D. Overview of strategies for overcoming the challenge of antimicrobial resistance. Exp Rev Clin Pharmacol 2010;3:667-686.

6. Johnson AP. Methicillin-resistant Staphylococcus aureus: the European landscape. J Antimicrob Chemother 2011;66(Suppl. 4): iv43-iv48.

7. European Surveillance of Antimicrobial Consumption. ESAC yearbook 2008. Antwerp: University of Antwerp; 2010.

8. Borg MA. National cultural dimensions as drivers of inappropriate ambulatory care consumption of antibiotics in Europe and their relevance to awareness campaigns. J Antimicrob Chemother 2012;67:763-767.

9. Harbarth S, Monnet D. Cultural and socioeconomic determinants of antibiotic use. In: Gould IM, Van Der Meer J, editors. Antibiotic policies: fighting resistance. New York: Springer; 2007. p. 29-40.

10. Hofstede G, Hofstede GJ, Minkov M. Cultures and organisations: software of the mind. New York: McGraw-Hill; 2010.

11. European Centre for Disease Prevention and Control. Antimicrobial resistance surveillance in Europe 2010. Annual report of the European Antimicrobial Resistance Surveillance Network (EARSNet). Stockholm: ECDC; 2011.

12. Hofstede G. Culture's consequences: comparing values, behaviors, institutions and organizations across nations. 2nd ed. Thousand Oaks, CA: Sage Publications; 2001.

13. Hansen S, Schwab F, Asensio A, et al. Methicillin-resistant Staphylococcus aureus (MRSA) in Europe: which infection control measures are taken? Infection 2010;38:159-164.

14. MacKenzie FM, Bruce J, Struelens MJ, et al. Antimicrobial drug use and infection control practices associated with the prevalence of methicillin-resistant Staphylococcus aureus in European hospitals. Clin Microbiol Infect 2007;13:269-276.

15. Bronzwaer S, Lönnroth A, Haigh R. The European Community strategy against antimicrobial resistance. Euro Surveill 2004;9: 30-34. 
16. Allegranzi B, Bagheri Nejad S, Chraiti MN, et al. World Health Organization guidelines on hand hygiene in healthcare: a summary. Geneva: World Health Organization; 2009.

17. Dellit TH, Owens RC, McGowan Jr JE, et al. Infectious Diseases Society of America and the Society for Healthcare Epidemiology of America guidelines for developing an institutional program to enhance antimicrobial stewardship. Clin Infect Dis 2007;44:159-177.

18. Sawyer M, Weeks K, Goeschel CA, et al. Using evidence, rigorous measurement, and collaboration to eliminate central catheterassociated bloodstream infections. Crit Care Med 2010;38: S292-S298.

19. Wassenberg MW, de Wit GA, van Hout BA, Bonten MJ. Quantifying cost-effectiveness of controlling nosocomial spread of antibioticresistant bacteria: the case of MRSA. PLoS One 2010;5:e11562.

20. Nicol PW, Watkins RE, Donovan RJ, Wynaden D, Cadwallader H. The power of vivid experience in hand hygiene compliance. J Hosp Infect 2009;72:36-42.

21. Wilson J, Guy R, Elgohari S, et al. Trends in sources of meticillinresistant Staphylococcus aureus (MRSA) bacteraemia: data from the national mandatory surveillance of MRSA bacteraemia in England, 2006-2009. J Hosp Infect 2011;79:211-217.

22. Jarlier V, Trystram D, Brun-Buisson C, et al. Curbing methicillinresistant Staphylococcus aureus in 38 French hospitals through a 15-year institutional control program. Arch Intern Med 2010;170:552-559.
23. Magiorakos AP, Leens E, Drouvot V, et al. Pathways to clean hands: highlights of successful hand hygiene implementation strategies in Europe. Euro Surveill 2010;15:19560.

24. Köck R, Becker K, Cookson B, et al. Methicillin-resistant Staphylococcus aureus (MRSA): burden of disease and control challenges in Europe. Euro Surveill 2010;15:19688.

25. Wyllie DH, Walker AS, Miller R, et al. Decline of meticillinresistant Staphylococcus aureus in Oxfordshire hospitals is strain-specific and preceded infection-control intensification. BMJ Open 2011;1:e000160.

26. Ferguson JK. Preventing healthcare-associated infection: risks, healthcare systems and behaviour. Intern Med J 2009;39: 574-581.

27. Boyd S, Aggarwal I, Davey P, Logan M, Nathwani D. Peripheral intravenous catheters: the road to quality improvement and safer patient care. J Hosp Infect 2011;77:37-41.

28. Borg MA, Benbachir M, Cookson BD, et al. Health care worker perceptions of hand hygiene practices and obstacles in a developing region. Am J Infect Control 2009;37:855-857.

29. Gould DJ, Hewitt-Taylor J, Drey NS, Gammon J, Chudleigh J, Weinberg JR. The CleanYourHandsCampaign: critiquing policy and evidence base. J Hosp Infect 2007;65:95-101.

30. Berenholtz SM, Pronovost PJ, Lipsett PA, et al. Eliminating catheter-related bloodstream infections in the intensive care unit. Crit Care Med 2004;32:2014-2020. 